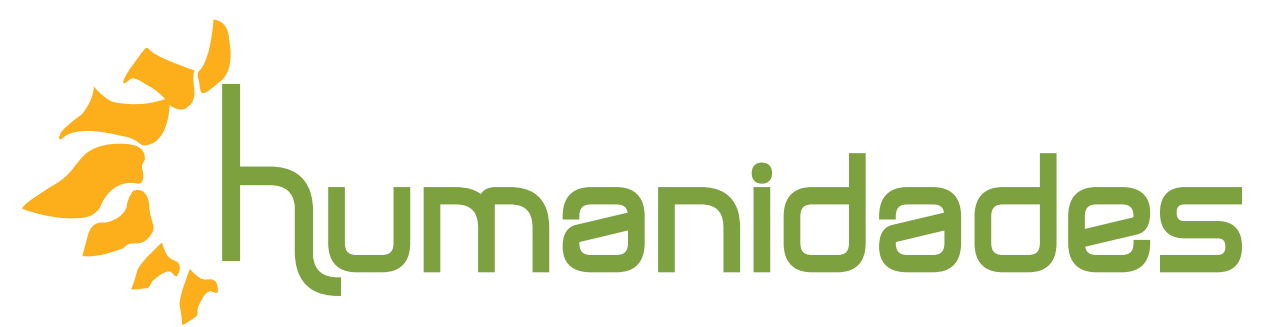

Revista humanidades

Enero-Junio, 2015 • Volumen 5, Número 1 • ISSN 2215-3934・pp. 1-23

\title{
La imagen de Estudios Generales y la calidad de gestión. Un modelo de análisis multivariable
}

DOI: http://dx.doi.org/10.15517/h.v5i1.19388

Recibido: 02-Diciembre-2014 / Aceptado: 26-Marzo-2015

\section{José Alberto Rodríguez Bolaños}

Doctor, profesor en la Escuela de Estudios Generales de la Universidad de Costa Rica

Correo electrónico: jar@demoscopia.co.cr 


\section{La imagen de Estudios Generales y la calidad de gestión. Un modelo de análisis multivariable}

\section{Resumen}

El artículo versa sobre el diseño, fundamentación teórica y aplicación práctica del modelo de análisis multivariable o factorial para medir la calidad de atención al cliente (estudiantes de estudios generales), por parte del equipo docente. El artículo se divide en tres partes: la primera es una introducción teórica sobre la calidad del servicio, mejoramiento continuo, la importancia del dato estadístico y la evaluación sistemática. La segunda parte es una reflexión sobre los métodos cuantitativos y cualitativos, y su importancia en la medición de los procesos sociales, en particular sobre el modelo que he diseñado para la Escuela de Estudios Generales. En la tercera parte se explican las características del modelo, su fundamentación estadística en lo concerniente a las muestras de los dos estudios ya realizados, así como los antecedentes preparatorias al estudio. En esta parte, además, se analizan algunos datos estadísticos que expresan los indicadores de gestión, según los factores de estudio, sus variables, acorde a cada una de las secciones de Estudios Generales y de la Escuela en general.

\section{A Multivariate analysis model. The image of General Studies and management quality.}

\footnotetext{
Abstract

The article focuses on the design, theoretical foundation and practical application of the model of multivariate or factorial analysis to measure the quality of customer service (general studies students) by the faculty. The article is divided into three parts: the first is a theoretical introduction on service quality, continuous improvement, the importance of statistical data and systematic evaluation. The second part is a reflection on the quantitative and qualitative methods, and its importance in the measurement of social processes, in particular about the model I designed for the School of General Studies. In the third part the technical characteristics and the statistics of the multivariable model are explained, as well as the variables and factors of the study, the comparative results in terms of management indicators by factor and variables for the School and its divisions.
}

\author{
Palabras claves: \\ Modelo de análisis \\ multivariable o factorial, \\ Calidad de la educación, \\ proceso educativo, Escuela \\ Estudios Generales, \\ Universidad de Costa Rica.
}


La educación de calidad es un derecho de todos y constituye el sexto objetivo de la agenda global de educación de la UNESCO, la Educación para Todos (EPT). Esta prioridad hace imprescindible evaluar la educación para monitorear el logro de dicho objetivo y también para perfeccionar políticas educativas que apunten a ofrecer una educación de calidad para todos. Los nuevos enfoques de la educación y las nuevas teorías, destacan la importancia de la calidad de la educación por sobre la cantidad. Incorporan, además, la noción de competencia, en tanto "aquello que produce un rendimiento superior en el trabajo" (McClelland, 1973). La calidad entendida más que como un índice o un número, conduce al mejoramiento continuo y sostenido, y hace que la evaluación del quehacer educativo sea vital. En ese sentido, la medición sistemática favorece este propósito.

Temas como la calidad referida a la formación de profesores, la inversión en infraestructura, la tecnología utilizada, la idoneidad, las relaciones humanas, la coherencia institucional, la actualización de los currículos, entre otros, son centrales y no deben de ser descuidados. Sin embargo, hoy se destaca un nuevo enfoque que agrega una dimensión por competencias y una organización institucional articulada en función de esa perspectiva, para construir sistemas educativos de calidad y que se asocien a las teorías del capital humano y del capital social.

Cuando se mencionan los sistemas de calidad en educación, se habla de una modernización que implica sistemas nacionales e internacionales de acreditación, estándares de calidad para práctica profesional, educación continua a distancia, postgrados, entre otros.

Implementar sistemas de calidad en instituciones de educación superior es complejo. Así, la evaluación y la acreditación de estándares de calidad en educación nacen debido y en confrontación a la masificación de la educación superior y su heterogeneidad. Hay una necesidad de generar una diferenciación 
institucional que promueva, en condiciones de mercado, los desarrollos competitivos de instituciones, los cuales den garantía a sus graduados, alejándose y marcando la diferencia con instituciones inescrupulosas que, en función de un lucro, ofertan servicios por debajo de los estándares mínimos.

La lógica del siglo XX y XXI ha sido la autonomía, el aumento de la cobertura de la educación superior y una explosión descontrolada en los ámbitos latinoamericanos de una oferta de dudosa calidad. Lo anterior, y una educación superior supeditada a las condiciones del mercado, enfrentan a las instituciones en el siglo XXI a la necesidad de generar procesos prístinos de evaluación competitiva que permitan lidiar con el exceso de una oferta profesional en un mercado masificado.

En nuestro país enfrentamos una diversidad de la calidad a todo nivel, pero sobre todo en el sector privado. Instituciones como la Universidad de Costa Rica (UCR) no se pueden dar el lujo de ofertar en este mercado, sin hacer un esfuerzo por continuar siendo los garantes de la calidad, la excelencia académica y una formación profesional del mejor nivel.

Hablar de evaluación, acreditación y certificación; implica hablar de mejorar, una mejora constante y sostenible. Si bien es cierto la educación no es un objeto y difiere mucho de los procesos de manufactura, los modelos de calidad continua y certificación en el ámbito privado pueden enseñarnos mucho acerca de cómo mejorar la calidad del servicio que presta la Escuela de Estudios Generales y la Universidad al país, a saber, una educación de calidad, eficiente, oportuna y a tono con las necesidades y demandas de un país en desarrollo.

El objetivo de la educación no puede estar basado en estándares mínimos que nos permitan obtener una acreditación de una carrera por parte de un ente especializado. 
El objetivo de la educación debe ser la excelencia, la mejor calidad posible, esto solo es factible en una lógica de mejoramiento continuo. El locus crítico de este proceso no debe estar solamente enmarcado en un agente acreditador externo, sino en una preocupación genuina de la institución a gestar dentro de sus relaciones intraorganizacionales, los procesos de autocrítica y transformación, los cuales le permitan seguir esta lógica de ofertar siempre lo mejor de lo mejor. Es por eso, y bajo esta filosofía, que se desarrolla en este trabajo un modelo de evaluación multivariable; un proyecto denominado "Sistema de evaluación de la imagen de Estudios Generales y la calidad de gestión". Su principio fundamental es medir para mejorar.

¿Qué es mejora? "Mejora” significa la creación organizada de un cambio beneficioso. El logro de niveles de rendimiento sin precedentes. Representa un sinónimo de "ruptura", es una necesidad de revisar, repensar y abandonar los modelos y paradigmas tradicionales de hacer las cosas, para así poder establecer la "divergencia". Es aplicable al concepto de calidad. En este sentido la "mejora continua" es acción constante y actividad recurrente para aumentar la capacidad para cumplir los requisitos; es un proceso que siempre se debe fundamentar en el principio de medir. Como señala Edwards Deming ${ }^{1}$ en su análisis sobre "Los catorce puntos y siete pecados mentales", que corresponden a los principios fundamentales de cómo lograr la calidad total (TQM); en el punto quinto señala que se debe mejorar constantemente, y por siempre, los sistemas de producción, servicio y planeamiento de cualquier actividad. En su planteamiento, la medición estadística (y también cualitativa) muestra con referentes empíricos, el cómo está funcionando la estructura organizacional (en este caso, la Escuela de Estudios Generales) y en qué medida se orienta hacia el mejoramiento continuo.

James Harrington $^{2}$ (1993 y 1997) definió la mejora continua como la acción de mejorar un proceso, cambiarlo para hacerlo más efectivo, eficiente y adaptable, 
Eduardo Deming ${ }^{3}$ (1996) propuso que la administración de la calidad total requiere de un proceso constante, que será llamado Mejoramiento Continuo, donde la perfección nunca se logra, pero siempre se busca. Su tesis la fundamentó en el principio de medir, evaluar, obtener datos objetivos como base para la toma de decisiones.

Como académicos, es preciso traducir estas filosofías industriales al delicado ámbito de la academia. Aunque esto suene a anatema, en ámbitos académicos, es una reflexión más necesaria que nunca. Ningún académico serio, sin importar su orientación filosófica, estaría en contra de abrir al interior de nuestras instituciones debates de revisión constante de los propios procesos de formación, para lograr formar, de la mejor manera, a quienes depositan en nosotros con confianza esta responsabilidad. Tal como se expone en un artículo publicado en la Revista humanidades $^{4}$, es necesario vencer complejos y prejuicios con respecto a medir, es necesario crear la cultura del dato, para obtener información y conocimiento, factores que permitan establecer procesos de autoevaluación e innovación, que faciliten la conquista de la excelencia académica.

Una filosofía de auto evaluación continua, basada en la reflexión y la autocrítica, contribuye a eliminar las debilidades y a afianzar las fortalezas de la organización, asimismo, genera una administración creativa. Se logra ser más eficiente, más comprometido y eficaz en un contexto dado, aún operando con recursos limitados.

El mejoramiento basado en la evaluación concentra el esfuerzo en ámbitos organizativos, administrativos y de procedimientos puntuales. Consigue mejoras a corto plazo y con resultados visibles. Trae consigo un uso más eficiente y austero de los recursos, genera una reducción en los costos y un aumento de la calidad del servicio prestado, el cual debe ser (siempre que sea posible) una meta en una institución que se financia con fondos públicos. 
La imagen de Estudios Generales y la calidad de gestión...

La mejora continua del servicio prestado en el desempeño global de una organización debería ser un objetivo permanente en esta.

Walter Shewart ${ }^{5}$ propuso, en la década de los chenta, un ciclo de mejora continua denominado PHVA (planificar, hacer, verificar, actuar), el cual luego fue popularizado por Eduard Deming. Sus principios son:

- Planificar: Establecer los objetivos y procesos necesarios para conseguir resultados de acuerdo con los requisitos del cliente y las políticas de la organización.

- Hacer: Implementar los procesos.

- Verificar: Realizar el seguimiento y la medición de los procesos y los productos respecto a las políticas, los objetivos y los requisitos para el producto, e informar sobre los resultados.

- Actuar: Tomar acciones para mejorar continuamente el desempeño de los procesos.

En consecuencia, los fundamentos orientadores de estos principios se fundamentan en los siguientes pilares

- Definir las metas

- Educar y entrenar

- Ejecutar la tarea (Recolectar datos)

- Verificar los resultados de la tarea ejecutada (recolectar información y generar datos)

- Actuar correctivamente en los procesos en función de los resultados

- Definir los métodos que permitan alcanzar las metas. 
Los principios y sus pilares se fundamentan en la información que emana de los clientes (estudiantes y profesores). El dato es el fundamento para evaluar y tomar decisiones.

El modelo de análisis diseñado para Estudios Generales, "Sistema de evaluación de la imagen de Estudios Generales y la calidad de gestión," tiene como referente teórico y modelo práctico estos principios, tal como se relacionará posteriormente en este artículo.

Diseñar y aplicar un modelo de análisis sobre la calidad del servicio, implica relacionar técnicas y métodos, conjugar sistemas de interpretación de la realidad.

\section{Fundamentos de} la técnica

Una investigación social de calidad es un acto de artesanía intelectual. En la investigación de públicos, grupos o sociedades en general, aunque puede ser respaldada por un proceso y una organización empresarial, siempre debe estar presente la relación del investigador con los segmentos de estudio, el análisis del dato, la ubicación de este en el proceso histórico y en el escenario sociocultural y político.

La artesanía intelectual como lo señalaba Mills, Charles W. ${ }^{6}$ requiere de imaginación. Debe huir del fetichismo del método y de la técnica, debe basarse en el principio de que cada investigador construya su teoría; que la teoría y el método sean permanentemente parte del ejercicio de un oficio. Cada problema de estudio requiere deseablemente la aplicación de una o más técnicas de estudio. No hay una fórmula única en la aplicación de las técnicas de investigación. Al igual que un artesano, cada investigador al inicio de un proyecto debe de escoger las herramientas que le permitirán cumplir a cabalidad con su propósito.

En consecuencia, es necesario evitar modas investigativas, aquellas que pretenden medir y analizar el mundo circundante con una reiterativa aplicación de técnicas 
La imagen de Estudios Generales y la calidad de gestión...

constituidas en fetiches, tal como lo señala Pitirim Sorokin ${ }^{7}$. Toda técnica tiene sus límites de aplicación, o sea, hay temas y problemas de estudio que pueden ser analizados por medio de una encuesta, hay otros que no soportan tal técnica, lo que obliga, como buenos artesanos intelectuales, a aplicar otros medios de recolección de información, acorde al problema en cuestión. Por ejemplo, técnicas cualitativas y proyectivas, o, las técnicas cuanti-cualitativas.

Desde el inicio mismo de las Ciencias Sociales, se ha planteado un dilema: ¿qué es lo verdaderamente medible cuantitativamente en la conducta humana? -y cuyos resultados sean generalizables a toda una población-, ¿qué aspectos no lo son? Se puede descubrir su esencia, construyendo a partir de ahí tendencias o esquemas interpretativos de la conducta y de la percepción humana, centrando el énfasis en la calidad o esencia del fenómeno.

Estas dimensiones del conocimiento, han favorecido la división de las técnicas de estudio en dos grandes categorías: lo cuantitativo y lo cualitativo.

Hablar de estas dos dimensiones de la investigación social, obliga a mencionar brevemente dos enfoques clásicos: el de Emile Durkheim y el de Florian Znaniecki ${ }^{8}$. Ambos definen con gran claridad estas dos tendencias. Por un lado, Durkheim en su libro Las Reglas del Método Sociológico ${ }^{9}$, señala que la primera regla y la más fundamental es considerar los hechos sociales como cosas. Todo objeto de ciencia es una cosa, o sea algo que puede ser medido estadísticamente. Esta visión establece un patrón de estudios empírico-estadístico, cuyos orígenes se ubican principalmente en la primera mitad del siglo XIX hasta la actualidad. La aplicación de este patrón de análisis se caracteriza por la búsqueda o construcción del "coeficiente estadístico".

Por otro lado, Florian Znaniecki, cuyos principales trabajos se ubican en la década de los treinta del siglo XX, reconoce el "valor" de los estudios cuantitativos, pero 
mantiene la posición de que para un investigador los datos de la cultura son siempre datos de "alguien" y nunca de "nadie". Los datos tienen un carácter esencial, él lo denomina "coeficiente humanístico", o sea, el dato natural. El "coeficiente estadístico" expresa un dato de carácter cultural, o sea, un dato que pierde el sentido y el contenido personal.

El dato de "alguien", es un dato con sentido específico, humanizado por la expresión y el sentimiento de alguien concreto que expresa su particularidad, pero inmerso en un espacio sociocultural, o sea, su conglomerado social. A partir de estos autores, y otros más, la investigación siguió su curso con resultados positivos, fluctuando entre ambas posiciones, en sus inicios muy divergentes, pero que con el correr de la primera mitad del siglo XX, se constituyeron en tendencias de investigación complementarias, abandonando el sentido de exclusión de sus inicios. En la actualidad, una no desplaza a la otra, ambas se complementan, cada una tiene su espacio propio y una dimensión de acción compartida.

Estos principios han formado parte del modelo diseñado para la Escuela de Estudios Generales. Su preparación ha sido producto de entrevistas de profundidad, observación de modelos de evaluación y datos estadísticos derivados de encuestas. Se toman en cuenta muestras representativas, tanto las exploratorias, como aquellas inducidas bajo el propósito del modelo; se tiene como meta lo señalado en la introducción de este documento: crear mejora constante, acrecentar la calidad y revisar procesos.

Las técnicas cuantitativas se fundamentan en el principio de que el hecho social en estudio puede ser medido estadísticamente. Esto significa que la opinión pública, las percepciones humanas, las actitudes, los valores y otras manifestaciones humanas a nivel social, pueden ser expresados en cifras, cuadros estadísticos, gráficos y porcentajes. Estas expresiones conforman el "coeficiente estadístico". 
En el modelo de análisis se mide el hecho del conocimiento, del tipo de información que el cliente (estudiantes de la Escuela de Estudios Generales) tiene, y de la calidad de la información enviada o recibida. En la dimensión cuantitativa, se toma el dato colectivo, según las diferentes dimensiones de las mediciones, no en el hecho personalizado. Ya sea que se trate de estudios censales o de muestras de población (para el modelo de evaluación que nos ocupa han sido muestras), el principal interés estriba en medir las tendencias colectivas de la opinión o percepción de los hechos, o sea, el conjunto de acciones que comprenden "la calidad de la educación". De ahí que conceptos como "representativo", "significativo", "confianza del dato", "error", entre otros, sean claves en este proceso de medición y análisis.

El recurso de medir implica la aplicación de técnicas de estudio que garanticen que tal acto es totalmente factible, que es confiable y que puede ser repetido, con el propósito de comparar datos, establecer tendencias y observar cambios. Bajo estos intereses, la búsqueda del "coeficiente estadístico" es fundamental en el estudio de esta determinada área de la conducta, de la relación del estudiante, del profesor y de la Escuela de Estudios Generales. Observar, medir, comparar y experimentar son sus fundamentos; principio planteado por Augusto Comte, en los inicios de la sociología como disciplina científica.

Actualmente, las Ciencias Sociales, en general, brindan una amplia gama de técnicas de investigación cuantitativas. En el estudio "Sistema de evaluación de la imagen de Estudios Generales y la calidad de gestión" se han aplicado diversas técnicas cualitativas y cuantitativas. Durante la parte preparatoria, se aplicó un conjunto de 8 entrevistas de profundidad entre coordinadores de sección, además, entrevistas de consulta a nivel de la Dirección de la Escuela. De igual forma, se analizaron datos e instrumentos aplicados en otras mediciones. 
Complementariamente, se realizó una encuesta exploratoria, con una muestra de 848 estudiantes de la Universidad de Costa Rica y una prueba del instrumento, con una muestra estadística de 80.

Se han realizado dos evaluaciones. La primera, en el mes de junio del 2013, con una muestra de 841 ; y la segunda, en el mes de noviembre del mismo año, con una muestra de 780. Esta fase cuantitativa se debe combinar con fases cualitativas; se trata del momento en que, producto del análisis de los datos, los equipos de trabajo de cada sección analizan información estadística. De igual forma, determinan las causas de las calificaciones positivas y negativas, luego, convierten el dato general (antes llamado "dato de nadie") al "dato de alguien" (que es el dato de la sección); este, a su vez, se puede reducir a un dato más personalizado.

Lo anterior no es más que humanizar la información. Mejorar procesos, elevar la calidad a estándares superiores, aplicar el mejoramiento continuo (PHVA) y otros aspectos más, se deben fundamentar en los datos (coeficientes estadísticos), más los datos de la misión y la cultura organizacional de la Escuela de Estudios Generales (coeficientes humanísticos).

De acuerdo a lo comentado en los apartados anteriores, a continuación se analizarán los antecedentes del proyecto.

El conocimiento sobre la imagen que alumnos y profesores tienen sobre la

\section{Antecedentes y justificación del proyecto} Escuela de Estudios Generales y la capacidad de gestión, gira más en torno a los comentarios, suposiciones y observaciones corrientes, más que en estudios sistemáticos que parten de muestras e instrumentos adecuados para una evaluación idónea.

Bajo la orientación de lo señalado en la primera parte de este documento, una organización que brinda un servicio, cualquiera que sea su propósito, requiere conocer la percepción de sus clientes y de sus actores organizacionales. Como se 
La imagen de Estudios Generales y la calidad de gestión...

ha señalado anteriormente, la percepción bien medida se traduce en procesos de valoración, calificación y recomendaciones para un mejoramiento de la prestación del servicio. Lo anterior permite fundamentar procesos de cambio, corregir errores, contribuir a la búsqueda de la excelencia, entre otros.

El conocimiento debe inducir a la toma de decisiones. El dato o las bases de datos son fundamentales para eso. No obstante, el dato como elemento sustantivo en la toma de decisiones es parte de una cultura para la acción, la cual no siempre predomina en nuestras escuelas. Lo usual, como se ha señalado, es que la fuente $u$ origen de los datos proceda de medios comunes e improvisados, más apegados a la casualidad que a lo sistemático y sin fundamento de representatividad estadística.

Estudios Generales tiene antecedentes positivos en este campo. En el año 2004 se realizó un estudio que involucró a 1013 estudiantes de la Universidad de Costa Rica ${ }^{10}$. Los resultados de esta investigación fueron expuestos ante diversas autoridades de la Universidad.

En el año 2011, se realizó otra encuesta, esta vez en una población de 848 estudiantes de la Universidad de Costa Rica ${ }^{11}$. En ambos estudios participaron estudiantes del curso Fundamentos de Sociología. A partir de estas experiencias, se puede decir que es muy útil implantar, de forma sistemática, un análisis multivariable, el cual contribuya a generar un aporte significativo a la toma de decisiones. Es importante señalar que este modelo está enfocado en la perspectiva de marca y la calidad de atención al cliente.

Desarrollar un modelo de análisis multivariable que permita monitorear la imagen e identidad de marca de la Escuela de Estudios Generales, así como, la satisfacción sobre la calidad del servicio al cliente (estudiantes de la Escuela de Estudios Generales). Además, identificar áreas de deterioro y oportunidades de mejoramiento.

\section{Objetivo del análisis}


El modelo a realizar está basado en un análisis multivariable o factorial. A continuación, se exponen las características metodológicas de este modelo de análisis.

El análisis de factores es una técnica multivariada ideal para el análisis de información, cuando el propósito es determinar el comportamiento y grado de satisfacción de los clientes o públicos particulares, con respecto a un servicio. En este caso, el proceso educativo en la Escuela de Estudios Generales, mediante la construcción de índices e indicadores de evaluación.

Si se toma en cuenta que no todas las dimensiones (factores) involucradas son igualmente importantes, es posible desarrollar un modelo que pondere cada uno de esos factores y que exprese el resultado final en forma de un índice que servirá como agente de evaluación o imagen global.

El indicador de cada factor se calculará como la suma ponderada de las notas obtenidas en cada variable, tal y como lo muestra la siguiente fórmula:

Donde:

$$
I_{5}=\sum_{i=1}^{k} P^{*} w_{i}
$$

$\mathrm{P}_{\mathrm{i}}=$ Identifica la nota de cada pregunta

$\mathrm{W}_{\mathrm{i}}$ = Corresponde al factor de ponderación de cada pregunta

k= Número de preguntas de cada dimensión o factor 
La imagen de Estudios Generales y la calidad de gestión...

El indicador global se calculará como la suma ponderada de los indicadores de cada dimensión o factor.

$$
g=\sum_{i=1}^{h} I s_{i} t_{t}
$$

Donde:

$\mathrm{Ig}=$ Indicador global

$\mathrm{Is}_{1}=$ Identifica el indicador de cada dimensión

$\mathrm{t}_{\mathrm{i}}=$ Corresponde al factor de ponderación de cada dimensión

Para realizar este cálculo es necesario establecer pesos a cada factor, de tal manera que los puntajes no se asignen equitativamente, sino ponderados de acuerdo con el grado de valoración o importancia. En el caso de las dos mediciones realizadas, los pesos por factor han sido los siguientes:

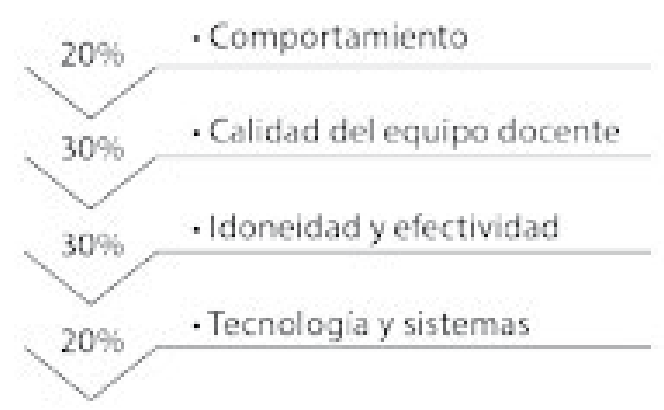

Figura 1: Pesos por factor

Fuente: Elaboración propia 
Tanto los factores como su peso son el producto de sesiones de trabajo con la Dirección de la Escuela y posteriormente probados entre la población objeto del estudio.

Las notas de cada pregunta, así como la del índice global, oscilará entre 0 y 10, donde 0 es la nota mínima y el 10 la nota máxima. Se pueden establecer valores negativos, si se desea una personalización mayor.

Además, la sistematicidad en las mediciones permitirá obtener a mediano plazo la comparación del comportamiento de los indicadores de gestión propuestos, establecer tendencias y poder construir supuestos explicativos, intra o extra institucional, que inciden sobre los espacios perceptivos y de la evolución de los actores sociales involucrados.

De acuerdo con los datos que se exponen a continuación, los productos a obtener son los siguientes:

- Indicador global a nivel de la Escuela de Estudios Generales, a partir del tipo de estudio.

- Indicador por cada factor, según la calificación de los estudiantes.

- Indicador por variable.

Estos indicadores se podrán cruzar con las variables sociodemográficas y otras características propias del estudio y de las poblaciones. 
La imagen de Estudios Generales y la calidad de gestión...

Los siguientes son los tamaños de muestra según el tipo de población de estudio en las mediciones de junio y noviembre del 2013:

\begin{tabular}{|l|l|l|l|l|}
\multirow{2}{*}{\multicolumn{1}{c|}{ Escuela }} & \multicolumn{2}{c|}{ 1ra. Medición } & \multicolumn{2}{c|}{ 2da. Medición } \\
\cline { 2 - 6 } & Muestra & $\begin{array}{l}\text { Margen } \\
\text { de error }\end{array}$ & Muestra & $\begin{array}{l}\text { Margen } \\
\text { de error }\end{array}$ \\
\hline Seminario de Realidad Nacional & 134 & $\pm 8,5 \%$ & 110 & $\pm 9,3 \%$ \\
\hline Problemas Ecológicos & 71 & $\pm 11,6 \%$ & 99 & $\pm 9,8 \%$ \\
\hline $\begin{array}{l}\text { Introducción al Pensamiento Lógico } \\
\text { Matemático, Fundamentos de Física }\end{array}$ & 105 & $\pm 9,5 \%$ & 105 & $\pm 9,5 \%$ \\
\hline $\begin{array}{l}\text { Reproducción, Sexualidad y } \\
\text { Humanismo }\end{array}$ & 60 & $\pm 12,7 \%$ & 76 & $\pm 11,2 \%$ \\
\hline Arte & 134 & $\pm 8,5 \%$ & 117 & $\pm 9,0 \%$ \\
\hline Humanidades & 246 & $\pm 6,3 \%$ & 213 & $\pm 6,7 \%$ \\
\hline Fundamentos de Sociología & 91 & $\pm 10,3 \%$ & 60 & $\pm 12,5 \%$ \\
\hline Muestra total & 841 & $\pm 3,4 \%$ & 780 & $\pm 3,5 \%$ \\
\hline
\end{tabular}

Figura 1: Pesos por factor

Fuente: Elaboración propia

Las muestras por sección varían de acuerdo con el universo de cada uno de estos. Dada la diferencia de población entre las secciones, no es posible estandarizar la muestra. Se aplica la proporcionalidad, de acuerdo con el peso que cada sección tiene dentro del universo de Estudios Generales.

El gráfico siguiente representa el resumen de evaluaciones para la Escuela de Estudios Generales. 


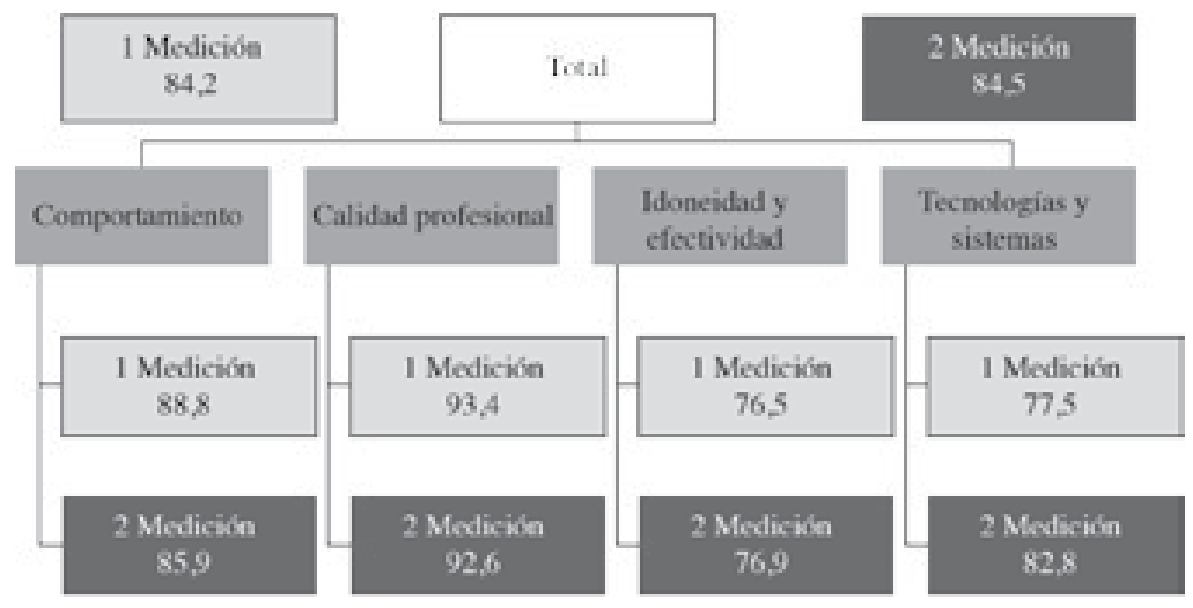

Figura 3: Evaluaciones de la Escuela de Estudios Generales Fuente: Elaboración propia

Como se puede observar, el indicador general tiene leves diferencias entre la primera y segunda evaluación. Si la analizamos de acuerdo con los factores, hay una leve disminución en los factores comportamiento y calidad profesional. Para el factor "idoneidad y efectividad" el aumento es mínimo. Tecnología y sistemas es el factor de mayor crecimiento. Las áreas críticas se concentran en el factor "idoneidad y efectividad". El factor "tecnología y sistemas" presenta mejoría, sin llegar a constituirse en fortaleza.

Derivado de este enfoque general y de acuerdo con el espíritu de este modelo, en la Escuela de Estudios Generales es preciso formular metas y objetivos en cada sección, cuyo propósito sea mejorar en cada uno de los factores. El modelo permite identificar en cuáles variables de cada factor se presentan las mayores fortalezas y debilidades. El trabajo de "mejora" hacia la excelencia requiere de un trabajo de conjunto. El objetivo no es sostener los indicadores, sino mejorarlos. 
La imagen de Estudios Generales y la calidad de gestión...

Si analizamos los indicadores por sección, podemos observar los cambios entre ambas mediciones.

El objetivo práctico de este modelo es que cada sección debe identificar sus áreas débiles, fortalezas y formular su metas. Con lo anterior será posible llegar hasta la identificación de elementos críticos a nivel personal, corregir errores para mejorar los indicadores de cada factor y por ende de sus variables.

Este modelo permite medir el crecimiento, el estancamiento y el deterioro; todo esto

Escuela Estudios Generales a nivel general de la Escuela, a nivel de cada sección, a nivel de cada factor y de sus variables. Todo estancamiento se debe ver como estado de situación negativa.

\begin{tabular}{|c|c|c|c|c|c|c|c|c|c|c|}
\hline \multirow{4}{*}{ Sección } & \multicolumn{8}{|c|}{ Factor } & \multirow{2}{*}{\multicolumn{2}{|c|}{ Total }} \\
\hline & \multicolumn{2}{|c|}{ Comportamiento } & \multicolumn{2}{|c|}{$\begin{array}{l}\text { Calidad } \\
\text { profesional }\end{array}$} & \multicolumn{2}{|c|}{$\begin{array}{l}\text { Idoneidad y } \\
\text { efectividad }\end{array}$} & \multicolumn{2}{|c|}{$\begin{array}{l}\text { Tecnología y } \\
\text { sistemas }\end{array}$} & & \\
\hline & \multicolumn{2}{|c|}{ Medición } & \multicolumn{2}{|c|}{ Medición } & \multicolumn{2}{|c|}{ Medición } & \multicolumn{2}{|c|}{ Medición } & \multicolumn{2}{|c|}{ Medición } \\
\hline & 1 & 2 & 1 & 2 & 1 & 2 & 1 & 2 & 1 & 2 \\
\hline $\begin{array}{l}\text { Fund. Física y lógico } \\
\text { matemático }\end{array}$ & 92.8 & 83.1 & 94.3 & 93.5 & 78.4 & 73.9 & 77.9 & 86.6 & 85.8 & 84.2 \\
\hline Realidad Nacional & 87.3 & 87.4 & 92.9 & 91.9 & 77.8 & 76.5 & 81.3 & 75.9 & 84.8 & 82.9 \\
\hline Probl. Ecológicos & 79.2 & 82.8 & 92.1 & 89.7 & 75.7 & 77.5 & 81.2 & 86.8 & 82.0 & 84.2 \\
\hline Sexualidad- Humanismo & 93.1 & 85.3 & 93.5 & 89.7 & 80.3 & 75.2 & 88.1 & 89.6 & 88.7 & 84.9 \\
\hline Arte & 89.2 & 89.3 & 94.4 & 95.0 & 74.8 & 81.1 & 74.0 & 81.7 & 83.1 & 86.7 \\
\hline Humanidades & 90.1 & 85.1 & 95.1 & 92.7 & 75.6 & 75.5 & 73.1 & 72.6 & 83.4 & 81.4 \\
\hline Sociología & 86.0 & 91.5 & 91.6 & 94.7 & 74.0 & 81.3 & 73.7 & 82.5 & 81.3 & 87.5 \\
\hline Indicador global & 88.3 & 86.3 & 93.4 & 92.4 & 76.7 & 77.2 & 78.4 & 82.2 & 84.2 & 84.5 \\
\hline
\end{tabular}

Figura 4: Indicadores y resultados de la Escuela de Estudios Generales Fuente: Elaboración propia 
El siguiente cuadro-resumen muestra, a nivel de sección, los indicadores de cada factor y el resultado a nivel global de la Escuela de Estudios Generales.

En el cuadro anterior se compara el estado de situación de cada sección, según cada factor y su proceso de mejoramiento, estancamiento o deterioro de una medición a otra.

\begin{tabular}{|c|c|c|c|c|c|c|c|c|c|}
\hline Variables o aspectos evaluados & \multicolumn{2}{|c|}{ TOTAL } & 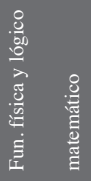 & 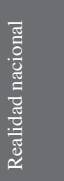 & 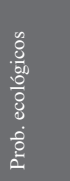 & 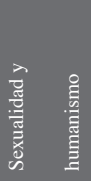 & 㷇 & 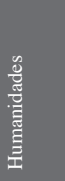 & $\begin{array}{l}\frac{\pi}{60} \\
\frac{0}{0} \\
\frac{0}{0} \\
0\end{array}$ \\
\hline \multirow{2}{*}{$\begin{array}{l}\text { 6. El profesor, ¿tiene o no una actitud de orden y } \\
\text { cumplimiento con los objetivos y propósitos del curso? }\end{array}$} & 1. Medición & 94,2 & 98,1 & 89,8 & 83,1 & 94,6 & 97,7 & 98,3 & 94,0 \\
\hline & 2. Medición & 92,0 & 99,0 & 85,6 & 89,7 & 82,4 & 97,3 & 91,7 & 98,3 \\
\hline \multirow{2}{*}{$\begin{array}{l}\text { 7. En este curso el profesor le ha explicado con claridad, } \\
\text { ¿qué es el humanismo y sus beneficios en su vida general } \\
\text { y a nivel profesional? }\end{array}$} & 1. Medición & 70,7 & 58,1 & 73,3 & 59,6 & 83,0 & 70,4 & 80,4 & 70,7 \\
\hline & 2. Medición & 70,8 & 52,3 & 83,0 & 70,1 & 71,4 & 69,6 & 71,3 & 77,9 \\
\hline \multirow[t]{2}{*}{ 8. En que grado el profesor planifica sus lecciones? } & 1. Medición & 79,1 & 85,1 & 77,4 & 76,3 & 83,9 & 79,8 & 79,7 & 72,0 \\
\hline & 2. Medición & 78,5 & 85,7 & 74,7 & 79,7 & 76,0 & 80,5 & 74,0 & 79,4 \\
\hline \multirow{2}{*}{$\begin{array}{l}\text { 9.Si usted tuviese que llevar algún curso y este profesor } \\
\text { lo imparte, ¿lo matricularía con él? }\end{array}$} & 1. Medición & 81,7 & 97,1 & 76,6 & 57,8 & 91,2 & 85,0 & 82,9 & 81,2 \\
\hline & 2. Medición & 82,0 & 88,2 & 82,9 & 66,3 & 76,8 & 89,2 & 76,0 & 94,9 \\
\hline
\end{tabular}

Figura 5: Tamaños de muestra según el tipo de población de estudio Fuente: Elaboración propia

Es interesante analizar la variable \#7. Esta se mantiene en 70.7 y 70.8 , respectivamente. Se presentan algunas variaciones, según las secciones de la Escuela, pero en general la medida es constante. Este es un caso, entre otros, en el cual el análisis debe generar una señal de advertencia, no solo es necesario detener la caída del indicador global del factor, sino que hay que trabajar de forma intensa en mejorar el indicador de esta variable. Lo anterior porque el humanismo, y sus beneficios en lo personal y profesional, es básicamente la razón por la que existe la Escuela de Estudios Generales. 
La imagen de Estudios Generales y la calidad de gestión...

Esta flexibilidad del sistema, permite apuntar hacia elementos muy específicos, identificar fortalezas y debilidades del sistema, realizar recomendaciones precisas, orientar acciones a nivel general y particular en la búsqueda de la excelencia. El modelo permite identificar cuáles "secciones" están en crecimiento, cuáles se están estancando y cuáles sufren deterioro; se dirige específicamente al punto crítico.

No se trata de medir por medir. Como se dijo al inicio de este documento, siguiendo a Mc Clelland, la calidad es entendida más que como un índice o un número, se trata de un camino de mejoramiento continuo y sostenido, donde es vital evaluar el quehacer educativo. En ese sentido, los postulados de W. Edwards Deming, J.Harrington, W Shewhart y otros autores citados, mencionan que hay que mejorar constantemente, y por siempre, los sistemas de producción, servicio y planeamiento de cualquier actividad. Así pues, la medición estadística, pero también la cualitativa, exponen, con referentes empíricos, el cómo está funcionando la estructura y el modelo organizacional de la Escuela de Estudios Generales y en qué medida se orienta al mejoramiento continuo.

La sistematicidad de la evaluación, la interpretación y uso de la información en la definición de metas y propósitos, convierten el dato estadístico en el dato de alguien y de algunos. Este es un proceso necesario de realizar a nivel de la Escuela de Estudios Generales, a nivel de cada sección, a nivel de cada docente y de cada equipo administrativo. 
1. Deming, W. Edwards. (2000). Out of the crisis. Cambridge: MIT Press.

2. Harrington, H. (1993). Mejoramiento de los procesos de la empresa. México: Editorial Mc. Graw Hill Interamericana, S.A.

3. Deming, W. Edwards (1993). The New Economics for Industry, Government and Education. Boston: MIT Press.

4. Rodríguez Bolaños, José Alberto. (2012). La cultura del dato. En Revista Notas humanidades. Disponible en: http://revistas.ucr.ac.cr/index.php/humanidades/ article/view/6450

5. Shewhart, Walter A. (1917). A study of the accelerated motion of small drops through a viscous medium. Lancaster: Press of the New Era Printing Company.

6. Mills, Charles Wright. (1961). La imaginación sociológica. México: Fondo de Cultura Económica.

7. Pitirim A. (1964). Achaques y manías de la sociología moderna y ciencias afines. España: Aguilar S.A.

8. Citado por Severiyn, Bruyn. (1972). La perspectiva humana en la sociología. Buenos Aires: XX editores.

9. Durkheim, É. (1986). Les Régles de la Méthode Sociologique. París: Presses Universitaires de France.

10. El margen de error fue de $\pm 3 \%$.

11. El margen de error fue de $\pm 3.4 \%$. Ambos estudios han sido analizados en la Revista humanidades. El trabajo se encuentra disponible en: http://revistas.ucr. ac.cr/index.php/humanidades/article/view/6450 
La imagen de Estudios Generales y la calidad de gestión...

Deming, W. (1993). The New Economics for Industry, Government and Education. Boston: MIT Press.

Deming, W. (1996). Out of the Crisis. Cambridge: MIT Press.

Deming, W. (2000). Out of the crisis. Cambridge: MIT Press.

Durkheim, Émile. (1986). Les Régles de la Méthode Sociologique. París: Presses Universitaires de France.

\section{Referencias bibliográficas}

Harrington, H. James. (1993). Mejoramiento de los procesos de la empresa. México: Editorial Mc. Graw Hill Interamericana, S.A.

Mills, Charles Wright. (1961). La imaginación sociológica. México: Fondo de Cultura Económica.

Pitirim A. Sorokin. (1964). Achaques y manías de la sociología moderna y ciencias afines. España: Aguilar S.A.

Rodríguez Bolaños, José Alberto. (2012). La cultura del dato. En Revista humanidades. Disponible en: http://revistas.ucr.ac.cr/index.php/humanidades/ article/view/6450

Shewhart, W. (1917). A study of the accelerated motion of small drops through a viscous medium. Lancaster: Press of the New Era Printing Company. 